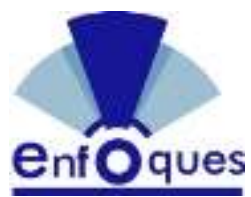

\section{ELEMENTOS DE LA PLANIFICACIÓN ESTRATÉGICA FINANCIERA EN LAS UNIDADES DE PLANES DE SALUD DE PDVSA- OCCIDENTE}

\author{
ELEMENTS OF FINANCIAL STRATEGIC PLANNING IN THE PDVSA- \\ OCCIDENTE HEALTH PLAN UNITS
}

WILMER CAMPOS

Artículo recibido diciembre 2019 | Arbitrado enero 2020 | Publicado 01 de abril 2020

\section{Resumen}

El objetivo fue identificar los elementos de la planificación estratégica financiera presentes en las unidades de planes de salud de PDVSA Occidente. La metodología fue descriptiva, no experimental, con un diseño de campo transeccional. La población quedó conformada por las clínicas industriales adscritas a las gerencias de salud de PDVSA Occidente. La información se recolecto a través de un cuestionario contentivo de 16 ítems, con escala de frecuencias. El mismo se validó por expertos y la confiabilidad calculada por la formula Alfa de Cronbach, obteniéndose 0,85. Para procesar los resultados se recurrió al método de la estadística descriptiva, en específico la media aritmética $(X)$. Se concluye que las actividades vinculadas al presupuesto, planes y estrategias financieras constituyen fortalezas. Paralelamente se detectó pocas acciones vinculadas con la visión, valores, objetivos y políticas financieras, representando leve fortalezas, además, son ejecutadas irregularmente las actividades correspondientes a la misión financiera reportando una debilidad

Palabras clave: Planificación; estrategias financiera, salud preventiva

\section{Wilmer Campos}

wilmer2606@hotmail.com Universidad del Zulia, Venezuela.

Licenciado en Administración, egresado de la Universidad del Zulia, Núcleo Costa Oriental del lago. Venezuela. Magíster Scientiarum en Gerencia de Empresas. Mención: Gerencia Financiera. Universidad del Zulia, Núcleo Costa Oriental del lago. Venezuela.

\begin{abstract}
The objective was to identify the elements of the financial strategic planning present in the PDVSA Occidente health plan units. The methodology was descriptive, not experimental, with a field and transectional design. The population was made up of the industrial clinics attached to the PDVSA Occidente health departments. The information was collected through a questionnaire containing 16 items, with a frequency scale. It was validated by experts and the reliability calculated by Cronbach's Alpha formula, obtaining 0.85 . To process the results, the descriptive statistics method was used, specifically the arithmetic mean $(X)$. It is concluded that the activities related to the budget, plans and financial strategies constitute strengths. At the same time, few actions related to the vision, values, objectives and financial policies were detected, representing slight strengths, in addition, the activities corresponding to the financial mission are reported irregularly, reporting a weakness
\end{abstract}

Key words: Planning; financial strategies, preventive health 


\section{INTRODUCCIÓN}

Debido a los cambios constantes en el entorno económico mundial, el proceso de la globalización de los mercados durante las últimas décadas, ha surgido la necesidad en las organizaciones empresariales de optimizar sus procesos gerenciales de manera estratégica, demandando calidad, eficiencia y equidad del sistema organizacional (Haime, 2013).

En el contexto de las transformaciones de las últimas décadas se pueden mencionar como tales los procesos de globalización, transformación de la economía mediante procesos intensivos en conocimiento, surgimiento de la sociedad de la información, nuevas situaciones asociadas a la gobernabilidad y a la ciudadanía, así como la potenciación de modelos de desarrollo basados en la competitividad internacional y las capacidades nacionales de crecimiento.

Se deduce entonces, lo importante para la gerencia de mirar la organización con una visión amplia, integradora, realista, humanista considerando las realidades culturales y las coyunturas de una organización en particular, en conjunción del manejo financiero. Con ello los gerentes buscan construir escenarios propicios para el logro de los objetivos organizacionales con base en la realidad existente.

En concordancia con lo anterior, las organizaciones y las personas cambian continuamente; estos cambios pueden ocurrir sea por las oportunidades surgidas o por las proyecciones según estrategias de desarrollo económico determinadas para cierto momento de la organización. El término desarrollo es aplicado cuando el cambio es intencional y proyectado como el proceso deliberadamente diseñado que debilite los efectos no deseados de este mismo cambio y potencie las posibilidades de crear futuro en la organización.
De tal manera, cuando se quiere llevar adelante un proceso de cambio se debe tener en cuenta las aspiraciones de las personas con la nueva situación, donde se les proporcione productividad, eficiencia y eficacia, con mayor aprovechamiento de los recursos.

Al respecto, el investigador, refiere que desde la gerencia financiera se pueden deliberar cambios favorables hacia el crecimiento organizacional ante nuevos esquemas de trabajo. En los mismos se deben introducir herramientas como la planificación financiera estratégica que permite a los gerentes y supervisores desarrollar una visión compartida de las prioridades y los desafíos de la organización, proporcionando una imagen clara de los costos de operación, inversión requerida y los impactos sobre las deudas, requiriendo una planificación para el flujo de caja suficiente para lograr el objetivo en el futuro.

Con ello se busca establecer la dirección de la organización, los objetivos de acuerdo con los valores fundamentales y las competencias de la organización, cuya aplicación está garantizada por la creación de un esbozo de los objetivos, especificando cómo y cuándo, así como las responsabilidades sobre la organización y ejecución de diversas tareas. La planificación estratégica financiera es realizada por los asesores financieros y en algunos casos por grupos de asesores financieros y sus asistentes, el asesor ayudará entonces a los clientes a implementar diferentes estrategias que hayan sido desarrolladas en el plan para alcanzar sus metas financieras (MacLeod, León, y Esquivias, 2011).

A este respecto, Magiolo, González, Bastidas y Ferrer (2014), consideran que muchas son las organizaciones que bien al nacer plantean sus estrategias 0 al enfrentarse a cambios radicales reorientan 
las mismas, o peor aún, nunca se plantean una planificación estratégica para orientar la toma de decisiones, así como la elaboración de la información necesaria, dificultando la preparación de los presupuestos anuales contrastando con cualquier esfuerzo de modernización. Este es el caso de la gestión de salud, deteniendo el impulso hacia una gestión eficiente de los recursos.

Con respecto a ello, Haime (2013) expresa la importancia y la necesidad de la planificación estratégica financiera en las instituciones de salud, comprendiendo procesos definidos que implican el análisis del entorno del negocio, apuntando hacia las oportunidades y amenazas, así como el análisis de la unidad económica en cuestión desde sus debilidades y fortalezas, para enfocar el posicionamiento estratégico, estrategias de negocios, planes de acción que desemboquen en el presupuesto y su control, evaluación y objetivos institucionales con un proceso de retroalimentación continuo.

Así, bajo la denominación de planificación estratégica financiera, para Galeano y Tinjacá (2012) se entiende como el proceso de conformación de varios escenarios futuros, permitiendo predecir diferentes situaciones positivas, negativas o estables ante lo cual se fijan posiciones de actuación al corto, mediano y largo plazo teniendo en consideración varios factores que podrían ser fluctuantes en el tiempo como la inflación, los tipos de cambio, las tasas de interés y todos aquellos que se necesitan para realizar la proyección financiera y que pueden tener una influencia determinarte en la economía interna de la empresa, teniendo como función la identificación de los objetivos de la empresa, realizar informes acerca de la situación real de la empresa en comparación a periodos anteriores con el fin de establecer estrategias para lograr los objetivos y conseguir el futuro deseado de la compañía.

Por su parte, MacLeod, León y Esquivias (2011) refieren a la planificación estratégica financiera como prioridades o direcciones que adopta la organización tras un análisis de las fortalezas, oportunidades, debilidades y amenazas en las que se encuentra inmersa, conformando un plan estratégico contentivo de proyecciones financieras de las necesidades y capacidades de recursos disponibles, que de manera operativa se refleja en el presupuesto anual, todo ello en función de dar cumplimiento a la misión y objetivos organizacionales.

De esta manera, una empresa que no cuente con este tipo de planificaciones, carece de sus oportunidades ventajosas, pues contemplan las tendencias del mercado, fomentando la dirección participativa, el idealismo colectivo bajo directrices con líneas de acción para la toma de decisiones, complementando la intuición y el juicio directivo, motivando a la institución a pensar de modo innovador bajo el espíritu emprendedor y lo proactivo en la toma de decisiones. Con ello, según Cabreras (2006), posibilitan la gestión del cambio dada su flexibilidad, así como las revisiones y controles periódicos.

Esta realidad no es ajena a las organizaciones de salud venezolanas. En este contexto, la empresa matriz Petróleos de Venezuela Sociedad Anónima (PDVSA), con una gran cantidad de empleados y extensión geográfica, así como la inversión, estructurada por diversas divisiones y a su vez por gerencias, no escapa a la necesidad de adaptarse a los cambios tantos internos como externos que ha experimentado en las últimas dos décadas, con políticas de gobierno entre las cuales se pueden mencionar el crecimiento y apertura a la sociedad venezolana desde la gestión social, 
aumentando el número de empleados así como de empresas de servicios, según se expresa en (PDVSA, 2019).

Una de las gerencias donde se refleja este aumento de personas es en las gerencias de salud de PDVSA Occidente, las cuales surgen para beneficio de sus trabajadores(as), familiares y jubilados a fin de brindar servicios de promoción de la salud, calidad de vida, prevención de enfermedades, tratamiento y rehabilitación de acuerdo con PDVSA (2019). A estas, se les cataloga como unas gerencias de apoyo dentro de la corporación, por servir como pináculo fundamental en la satisfacción del personal activo, jubilado y comunidad en general, entendiendo la salud como un valor corporativo, lo cual repercute positivamente en el incremento de la calidad de vida, productividad personal, familiar y laboral.

Sobre este particular en las gerencias de salud PDVSA Occidente, a través de entrevistas diagnósticas no estructuradas, observación directa, así como mediante la revisión de algunos trabajos realizados en este ambiente, el investigador ha inferido deficiencias en los estados financieros previstos, existiendo diferencias entre lo planeado y la situación en un punto del tiempo, creando desviaciones más allá de lo manejado dentro de los márgenes de error comunes; así mismo, se evidencia sub utilización de las inversiones $y$ financiamientos. Esta situación despierta la inquietud a nivel gerencial de las unidades de salud en la búsqueda de soluciones capaces de encauzar a un desempeño eficiente de cada uno de los entes financieros involucrados en el servicio de salud en PDVSA Occidente.

Se entiende que el crecimiento de la población a ser atendida por las gerencias de salud PDVSA Occidente, sin tomar en cuenta un plan estratégico para prever el actual escenario, ha desencadenado esta serie de situaciones no deseadas, las cuales, de no ser atendidas dentro de un esquema técnico científico, pueden llevar al colapso de los servicios de salud en esta empresa. Ante este panorama, la planificación estratégica representa un elemento útil para la introducción de mejoras, para el máximo aprovechamiento de los estados financieros planeados, inversiones y financiamiento.

Al enmarcar, la planificación estratégica financiera, en las unidades de salud de PDVSA Occidente, es importante considerar que para el manejo de los planes de salud se debe contemplar la incorporación de lineamientos capaces de permitir el control y la optimización en la toma de decisiones gerenciales, con una visión clara del escenario financiero. Es importante señalar como para Cabreras (2006) tiene una orientación a mediano y largo plazo, donde la ejecución a corto plazo se basa en la formulación e implementación de objetivos específicos, acciones estratégicas, todo lo cual es producto de un análisis sistemático sustentado en la identificación de las amenazas, oportunidades, debilidades y fortalezas.

De modo que se debe entender el entorno de evolución dinámico en donde se encuentra expuesta dicha gerencia, así como la manera en la cual sus lineamientos se ven afectados continuamente ante los cambios experimentados por la organización. Estos han sido introducidos por la globalización, nuevas tecnologías, internet, además de todo lo anteriormente expuesto, dando lugar de manera relevante a la necesidad de introducir planes de acción para obtener en las unidades de salud de PDVSA Occidente una organización óptima con buen servicio y ambiente de trabajo.

En tal sentido, se pretende que la presente investigación se oriente hacia el 
análisis de la planificación estratégica financiera con miras a la mejora de la gestión financiera, desembocando en respuestas eficaces y eficientes para con las necesidades asociadas al aseguramiento de la calidad en las unidades de planes de salud de PDVSA Occidente. Todo ello tiene como finalidad el guiar de manera estratégica al personal a resolver situaciones previsibles y a su vez con flexibilidad para atender casos fuera de lo previsto.

En atención a esto, el estudio se enfocará en las clínicas adscritas a estas gerencias, las cuales forman parte de la iniciativa de la industria petrolera para reforzar los planes y servicios de la red del Sistema Público Nacional de Salud, cuya estrategia está dirigida a preservar y mejorar la salud de los participantes, basada en métodos y tecnologías científicamente soportados y socialmente aceptado. En esta óptica, el estudio se orienta identificar los elementos de la planificación estratégica financiera presentes en las unidades de planes de salud de PDVSA Occidente.

\section{Planificación estratégica financiera}

Weston (2006), plantea que la planificación estratégica financiera implica la elaboración de proyecciones de ventas, ingresos y activos tomando como base estrategias alternativas de producción y mercadotecnia, así como la determinación de los recursos que se necesitan para lograr estas proyecciones. Aunado a ello, Mendoza (2013), refiere que la planeación estratégica financiera se orienta a la caracterización de los objetivos de la empresa, plasmar informes sobre la situación real del flujo de dinero de la empresa con respecto a otros periodos anteriores con el objeto de lograr estrategias para alcanzar los objetivos y obtener el futuro deseado de la compañía.
De este modo, Galeano y Tinjaca (2012) expresan que este tipo de planificación implica primeramente una visión clara del negocio considerando como punto de partida la enunciación de objetivos, metas, y estrategias, llevando a la especificación de plazos en el tiempo, en función de medidas financieras de inversión, financiación, operación y creación de valor. Dicha actividad es de carácter prospectivo, contentivo de escenarios futuros, visualizando así situaciones de carácter favorable o desfavorable de manera que se puedan tomar medidas de actuación a tiempo, entre los indicadores comunes se encuentran, las tasas de interés, la inflación, los tipos de cambio, entre otros.

De lo expresado por los autores, el investigador considera que la planificación estratégica financiera es un enfoque objetivo y sistemático que permite a la empresa asumir una posición proactiva y no reactiva en el mercado que compite, ya que es mejor adelantarse a los acontecimientos que estar a la espera de lo que pueda suceder.

\section{Elementos de la planificación estratégica financiera}

Según Perdomo (2004), es considerada un proceso, descompone una secuencia de fases necesarias para la consecución de un plan, que deberá estar en consonancia con la estrategia general, solo que en este caso el plan se materializa en números concretos, atiende a la medición de todas las acciones de carácter financieras de la empresa, puesto que todo flujo real tiene una contrapartida en valor monetario que debe ser valorado por la función financiera, dado en función de sus elementos, de financiamiento, planes de inversión y plan presupuestario. 
En ese orden de ideas, para Ross y otros (2006), constituyen el conjunto de enunciados, que regulan la vida de las finanzas a corto y largo plazo, de tal modo, definen los aspectos de importancia, sobre ahorro e inversión. Los citados autores, consideran que en las organizaciones los elementos financieros equivalen a visión, misión, valores, objetivos, estrategias, políticas, planes y presupuesto, denominados principios por su calidad de elementos iníciales de las finanzas, principales y básicos, porque sobre esos se forma el edificio financiero de la empresa.

Atendiendo estas consideraciones, para el investigador los elementos de la planificación estratégica financiera conforman la filosofía de las empresas, implicando un ejercicio de reflexión, análisis sistemático, de valor y sentido, sobre las realidades financieras de la compañía, orientada a la comprensión a cómo llegar a conclusiones sobre la existencia de todos los diversos elementos de la realidad empresarial, interesándose por llegar a definir conceptos y principios en las partes coexistente en el negocio, estos elementos están referidos a visión financiera, misión financiera, valores financieros, objetivos financieros, estrategias financieras, políticas financieras, planes financieros $y$ presupuesto.

\section{Visión financiera}

Para Collins y Porras (2007), la visión debe tener dos elementos, la ideología central (lo que se busca preservar) y el futuro previsto (lo que se busca cambiar). La ideología central incluye una descripción de los valores y propósitos centrales de la organización y el futuro previsto que está planteado como la definición de los objetivos a largo plazo, estos últimos definidos por estrategias operativas.
Plantea Betancourt (2010), que es una visualización de cómo será el futuro de una organización, el puerto al que queremos llegar, es el deseo de una organización que se proyecta en el futuro. La visión debe proveer el camino a seguir, generar el entusiasmo necesario, ofrecer criterios claros para el éxito que generen confianza en el liderazgo de la gerencia. Así mismo, Serna (2010), plantea que la visión es la declaración amplia y suficiente de donde quiere que su empresa este dentro de 3 a 5 años, no debe estar expresada en números, comprometer y motivar a gerentes $y$ empleados y promover la pertenencia de la organización.

En el contexto especifico financiero, Acedo (2004), define la visión financiera como una manifestación indicativa hacia donde se dirige la empresa financieramente a largo plazo; qué ha de ser el futuro considerando los recursos tecnológicos, también las necesidades, expectativas de clientes, así como, la aparición de nuevas condiciones del mercado. De acuerdo con Münch (2008), es el enunciado del estado deseado en el futuro para la organización. Debe ser breve, fácil de captar y recordar e inspiradora.

El investigador fija posición congruente con los autores antes citados, ya que considera que la visión financiera señala el rumbo a seguir, proporciona dirección financiera de las empresas, es la cadena o lazo que une el presente con el futuro de la organización, alude a la identidad, unidad en propósitos e incluye a todos los miembros de la organización.

\section{Misión financiera}

Campbell y Nash (2007), definen una misión como aquella que incluye propósito, estrategia, valores, políticas y estándares de comportamiento; esos elementos antes 
mencionados se refuerzan entre sí. El objetivo de una declaración de la misión es determinar la esencia de la organización, lo cual viene dado por la definición del negocio de esta, tomando en cuenta un enfoque integral que considere a todos los actores y elementos claves dentro del mismo.

Al respecto, la declaración de la misión suele centrarse según Thompson y Strickland (2010) en decir quiénes somos y que hacemos, definir de manera general las capacidades, las actividades que realiza la empresa y el enfoque del negocio. Según David (2008), se ha comprobado que las empresas que usan declaración de misión muestran un $30 \%$ más de rendimiento en comparación con las que no la hacen.

Por su parte, Chiavenato (2009) establece que, en el fondo, toda organización se crea para cumplir la finalidad de ofrecer un producto o un servicio a la sociedad. Parte de esta finalidad la representa la misión que es la razón esencial de ser y existir del organismo, además de su papel en la sociedad. Es de suma importancia vigilar cual ha sido el propósito con que se elabora la misión ya que debe representar un compromiso real y no ajustarse a una moda.

Específicamente, la misión financiera, según Al respecto, Mitzberg y Quinn (2004), describe, los propósitos financieros generales establecidos por la organización, proporciona criterios fundamentales al análisis de la adecuada inversión de la empresa a largo plazo. Para Ortega (2008), es la declaración duradera de los objetivos financieros distintivos entre organizaciones. Cada organización con sus principios, valores, visión, filosofía de sus propietarios, colaboradores y grupo con que interactúa en el mercado.

En relación a la misión del departamento financiero Mazón y otros
(2003), mencionan que la misión es administrar y reflejar en los documentos apropiados y en los que exige la normativa vigente los movimientos económicos y monetarios, así como tratar de obtener el máximo beneficio de las finanzas de la empresa (siguiendo políticas y estrategias determinadas por parte de la dirección) y en consecuencia para los accionistas de la misma.

El investigador, considera que la misión describe, los propósitos financieros generales establecidos por la organización, proporciona criterios fundamentales al análisis de la adecuada inversión de la empresa a largo plazo, así como da importancia para el desarrollo del trabajo de investigación como son propósito, estrategia, valores, políticas y estándares de comportamiento.

\section{Valores financieros}

Para Francés (2006), los valores financieros, constituyen herramientas aplicadas como directrices gerenciales, ocupan un lugar relevante en las teorías y prácticas financieras adquiriendo relevancia en la administración empresarial. Al respecto, para Mitzberg y Quinn (2004), constituyen reglas o pautas mediante las cuales una compañía exhorta a sus miembros a tener comportamientos consistentes con su sentido de existencia (orden, seguridad y desarrollo).

De este modo, son propósitos a los cuales la organización y sus miembros deben dedicar su energía. Sobre este punto Bruna (2007), expresa como las organizaciones intervienen en la creación de valores financieros en el cliente interno. Es así como gerentes, y supervisores aplican herramientas motivadoras, de comunicación adecuada en función de educarlas en promover rendimiento, productividad en la 
inversión realizada en el trabajador por la empresa.

Considerando que los valores financieros de una organización deben ser claros y precisos, las empresas deben mantener y recordar a los trabajadores sus valores; de manera tal, que influyan en el comportamiento de los individuos, permitiendo el logro de los objetivos y la puesta en marcha de la planificación estratégica financiera. De lo expresado, el investigador considera que los valores financieros constituyen un valor agregado coyuntural, para el éxito de la organización, por tal motivo, el personal debe conocerlos y practicarlos

\section{Objetivos financieros}

Según David (2008), los objetivos son esenciales para el éxito de la organización porque establecen el curso, ayudan a la evaluación, producen sinergia, revelan prioridades, permiten la coordinación, asientan las bases para la planificación, organizan, motivan y controlan con eficacia. El establecimiento de los objetivos es una participación activa que debe conducir a la aceptación y al compromiso.

Los objetivos constituyen un mecanismo básico para evaluar a los gerentes, son el instrumento principal para la supervisión del logro a largo plazo, establecen las prioridades corporativas, de las gerencias y de los departamentos. En este orden de ideas, Serna (2010), los objetivos son los resultados globales que una organización espera alcanzar en el desarrollo y operacionalización concreta de su misión y visión. Para una organización un objetivo es una situación deseada que debe alcanzarse y se refiere a un tiempo futuro así que señala el norte a seguir de la empresa y establece las normas para las actividades de los miembros.
Para Chiavenato (2009), los objetivos representan los resultados que la empresa espera obtener, son fines para alcanzar, establecidos cuantitativamente y determinados para realizarse transcurrido un tiempo específico. Dos características primordiales que permiten diferenciarlos de cualquier otra etapa de la planeación son: Se establecen a un tiempo específico y se determinan cuantitativamente.

El autor anteriormente citado, expone que los objetivos financieros son conceptos muy comunes es la sociedad que refleja un estado de futuro deseado, el cual se trata de convertir en realidad, en la práctica no son más que los resultados específicos en un tiempo determinado. Mientras la misión define cual es el negocio de la organización y la visión ofrece una imagen de lo que se debe hacer a nivel organizacional, los objetivos definen resultados concretos en un plazo específico y los indicadores van midiendo los resultados alcanzados.

Para Vera (2005), un objetivo financiero es una situación deseada que la institución intenta alcanzar a largo plazo. De acuerdo con Burbano (2005), son puntos de convergencia para la utilización de los recursos financieros de la institución, como el porcentaje de dividendos, índice de endeudamiento total (apalancamiento). De igual forma, Cárdenas y Nápoles (2002), consideran que los objetivos financieros son lineamientos de la institución enmarcados en planear y sistematizar todas las actividades del rendimiento sobre la inversión, relación del capital del trabajo, rotación de las cuentas por pagar; entre otros, con el fin de establecer metas alcanzables.

Atendiendo a lo expuesto, el investigador considera que los objetivos financieros son fines o metas viables $y$ cuantificables que pretende alcanzar una empresa son lineamientos de la institución 
enmarcados en planear y sistematizar todas las actividades del rendimiento sobre la inversión, relación del capital del trabajo, rotación de las cuentas por pagar; entre otros, con el fin de establecer metas alcanzables.

\section{Estrategias financieras}

Gitman, (2013) refiere que las estrategias financieras se definen como las acciones o pasos seguir para el logro de los objetivos financieros $\mathrm{y}$, por ende, necesita ser organizada, planeada, supervisada $y$ dirigida, lo que orientará el sentido de la misma. Al respecto, como toda subestrategia, debe estar en consonancia con los planes generales de la entidad y tener en cuenta a los participantes internos y externos.

Del mismo modo, López (2012), refiere que las empresas necesitan financiamiento para llevar a cabo sus estrategias de operación, inversión y financiamiento, dichas estrategias permiten abrir más mercado, aumentar la producción, construir o adquirir bienes, hacer alguna otra inversión que la empresa vea benéfica para sí misma o aprovechar alguna oportunidad que presente el mercado. De este modo, el referido autor, expresa que la inversión es el factor principal que le da impulso a la empresa acorde a su necesidad, siempre en busca de la compra de activos u retorno de inversión que proporcionen una sólida base de flujo operativo de capital y permitan generar una sustentabilidad de operación que permita el desarrollo a largo plazo de los negocios.

Uno de los puntos importantes a analizar dentro de la administración financiera, en la visión de Zayas (2007) es la determinación de la liquidez empresarial mediante la correcta gestión de la empresa, esta toma como punto de partida determinadas estrategias básicas, las cuales están orientadas a lograr un equilibrio entre los flujos positivos, que se dan cuando las entradas de efectivo superan a las salidas, y los negativos es cuando las salidas superan a las entradas, de tal manera que la entidad consiga tener los fondos adecuados para el máximo provecho.

Considerando lo expuesto por los autores citados, el investigador posee una visión congruente sobre las estrategias financieras, las mismas tratan de obtener fondos, para lograr inversiones exitosas, que permitan adquirir bienes y servicios.

\section{Políticas financieras}

Para Vera (2005), políticas financieras son decisiones financieras de la alta gerencia en las organizaciones, dirigidas a controlar y mejorar determinados aspectos de las finanzas. En esta perspectiva de acuerdo con Burbano (2005), son una serie de principios y líneas de acción que guían el resultado financiero hacia el futuro. Por su parte Perdomo (2004), señala que las políticas financieras son reglas y principios generales que sirven de guía al pensamiento y acción de subordinados.

De igual forma Welsch y otros (2005), determinan que las políticas financieras definen aspectos claves dentro de la organización en cuanto al manejo de sus Ingresos y egresos que son de gran ayuda en la toma de decisiones. Seguidamente, Ortega (2008:9), define las políticas como "guías para orientar la acción. Son criterios, lineamientos generales para observar en la toma de decisiones sobre problemas que se repiten de forma periódica dentro de una organización". Mientras que Münch (2008:46), considera que las políticas "son criterios generales de ejecución que auxilian el logro de objetivos y facilitan la realización de las estrategias" 
Es así como las organizaciones deberán definir sus políticas organizacionales en la forma más conveniente, para aprovechar oportunidades que le brinda el entorno y de acuerdo con sus capacidades y recursos, mantener su competitividad (estrategia institucional), para lo cual se estructuran y coordinan, elementos de una determinada forma (estructura organizativa).

En tal sentido y considerando el caso que ocupa la presente investigación, el investigador considera que las políticas financieras a emplear, va de la par de otros elementos como la misión, visión, objetivo y valores de la organización; las mismas se deben adecuar a su funcionamiento interno de acuerdo con las exigencias del entorno para el logro de sus objetivos.

\section{Planes financieros}

Los planes financieros, según Catacora (2008) consiste en la elaboración de previsiones a medio y largo plazo, en un horizonte de 3-5 años. Al ser previsiones a más de un año, tienen un elevado grado de incertidumbre, pero no obstante es conveniente realizarlas para estar mejor preparado y dirigir con más precisión la empresa, al marcar los rumbos que debe tomar. Un posterior control, permitirá a través de las desviaciones, analizar y corregir las tendencias.

Con el plan financiero se trata de indagar la liquidez futura de la empresa, plasmándolo en un cuadro de Tesorería, que se elabora de la forma más oportuna para poner en evidencia sus componentes:

Como presupuesto de caja (a partir de las Cuentas Provisionales de Resultados). A partir de las cuantas provisionales de Resultados. (Saldo inicial de tesorería, cobros, pagos). El Plan de Financiación comprende los recursos propios y la financiación ajena a largo plazo de la empresa destinada, en general, a financiar el activo permanente y a cubrir un margen razonable del circulante; incluye también los ingresos a distribuir en varios ejercicios, acciones propias y otras situaciones transitorias de la financiación básica.

Se distinguen dos fuentes principales de financiación definidas en el Plan Económico Financiero: recursos propios y recursos ajenos. De lo expuesto, Perdomo (2004), los define como programas cronológicos cuantitativos en el tiempo y dinero, con el objeto de precisar el desarrollo de las actividades de la empresa.

Además, menciona que los planes financieros pueden ser correctivos; tendientes a asegurar el cumplimiento de los objetivos señalados de otros planes, en los casos de desviaciones señaladas por los mecanismos de control y tendientes a asegurar el cumplimiento de objetivos modificados, respecto a planes originales cuando las desviaciones denotadas por los mecanismos de control, se juzguen imposibles o incosteables de corregir total o parcialmente, por lo tanto es necesario o conveniente modificar las metas u objetivos originales.

Asimismo, señala el citado autor que los Planes financieros de desarrollo, los cuales son tendientes a lograr objetivos de expansión, penetración de mercado, desarrollo de nuevos productos para el mismo mercado, o desarrollo de nuevos mercados para los mismos productos, o bien una combinación de estos objetivos, y tendientes a lograr objetivos de diversificación nuevos productos para nuevos mercados.

Por consiguiente, el investigador considera que los planes financieros son programas que permiten garantizar el cumplimiento de objetivos previamente establecidos por la alta dirección de la organización, a propósito de preservar o mantener su posición en el mercado. 


\section{Presupuestos}

Según Gitman (2003) el presupuesto es un plan de operaciones y recursos de una empresa, que se formula para lograr en un cierto periodo los objetivos propuestos y se expresa en términos monetarios. En otras palabras, hacer un presupuesto es simplemente sentarse a planear lo que se quiere hacer en el futuro y expresarlo en dinero.

Por lo tanto, cuando se hace un presupuesto para una empresa, en realidad se está planeando a futuro. Entonces, surgen preguntas qué vender, qué se necesita hacer para lograrlo, cuánto se tiene que gastar $y$, cuánto es la ganancia en un periodo.

En la visión de Koontz y Weihrich (2004), un presupuesto es un enunciado de resultados esperados, expresado en términos numéricos. De hecho, el presupuesto financiero con frecuencia se conoce como plan de utilidades. Suele expresarse en términos financieros, pero también en horas/hombre, unidades de producto, horas máquina o cualquier otra unidad de medida de tipo numérico.

Al respecto, Münch (2008), lo define como un documento expresado en términos económicos financiero o no financiero, muestran la asignación de recursos para llevar a cabo los planes y actividades de la organización. Según Burbano (2005), es la expresión cuantitativa formal de los objetivos que se propone alcanzar la administración de la empresa en un periodo, con la adopción de estrategias necesarias para lograrlos.

Por lo expuesto, el investigador considera que el presupuesto es una herramienta de planificación, coordinación y control de funciones que presenta en términos cuantitativos las actividades a ser realizadas por las empresas del sector hotelero, con el fin de llevar a cabo de modo productivo sus recursos, para alcanzar determinadas metas u objetivos financieros.

\section{MÉTODO}

La metodología empleada fue de tipo descriptiva, no experimental, con un diseño de campo y transeccional. La población quedó conformada por las clínicas industriales adscritas a las gerencias de salud de PDVSA Occidente, en específico: Hospital Coromoto, San Francisco, Edificio Miranda, Centro Petrolero Torre Lama y Boscán, La Concepción, 5 de Julio, La Salina, Tía Juana, Muelle Patria Grande, Muelle Libertador, Campo Rojo, Lagunillas Sur, Lagunillas Norte, El Menito, Bachaquero, Mene Grande, EI Vigía, y San Lorenzo.

De tal manera, los sujetos informantes fueron los gerentes, asesores gerenciales, supervisores de cada unidad administrativa, de cada distrito de las Gerencias de Salud en PDVSA Occidente. La información se recolecto a través de la aplicación de un cuestionario contentivo de 16 ítems, con escala de frecuencias, para un total aproximado de 121 unidades informantes, al que se le realizó un muestreo probabilístico siendo el tamaño de la muestra estratificada extraída de 68 sujetos. El mismo se validó por cinco expertos y la confiabilidad calculada por la formula Alfa de Cronbach, obteniéndose 0,85 indicando muy alta confiabilidad.

Para procesar los resultados de la aplicación del cuestionario se recurrió al método de la estadística descriptiva, y se empleó las medidas estadísticas de tendencia central, en específico la media aritmética $(\bar{X})$ o promedio.Para tal efecto, el investigador diseñó un (1) baremo para el análisis de la media aritmética, tal como se muestra en el tabla 1. 
Tabla 1. Baremo para la interpretación de la media aritmética

\begin{tabular}{ccl}
\hline INTERVALO & CATEGORÍA & DESCRIPCIÓN \\
\hline $4.21-5,00$ & MUY ALTA PRESENCIA & $\begin{array}{l}\text { Si el nivel de presencia es muy alto o alto se } \\
\text { considera una fortaleza para la planificación } \\
\text { estratégica financiera }\end{array}$ \\
$3.41-4.20$ & $\begin{array}{l}\text { ALTA } \\
\text { PRESENCIA }\end{array}$ & $\begin{array}{l}\text { Si el nivel de presencia es moderado se considera } \\
\text { MODERADA }\end{array}$ \\
PRESENCIA & $\begin{array}{l}\text { una leve fortaleza para la planificación estratégica } \\
\text { financiera }\end{array}$ \\
$1.81-2.60$ & BRAJA & $\begin{array}{l}\text { Si el nivel de presencia es bajo o muy bajo se } \\
\text { considera una debilidad para la planificación }\end{array}$ \\
$1,00-1.80$ & MUY BAJAPRESENCIA & estratégica financiera
\end{tabular}

\section{RESULTADOS}

La tabla 1, muestra los resultados obtenidos sobre el indicador visión financiera perteneciente a la dimensión elementos de la planificación financiera, observándose un promedio de 2,89 ubicándola en la categoría de moderada presencia, señalando leve fortaleza. En este sentido, en opinión de los sujetos informantes, en las unidades de planes de salud donde ellos laboran, con moderada presencia se visualiza el futuro a través de criterios claros para el éxito $(3,38)$; no obstante con baja presencia las decisiones se toman considerando hacia donde se dirige la empresa financieramente en el futuro $(2,40)$.

Tabla 2. Visión financiera

\begin{tabular}{lcc}
\hline \multicolumn{1}{c}{$\begin{array}{c}\text { Ítems } \\
\text { En la unidad de planes de salud donde usted labora: }\end{array}$} & Media & Categoría \\
\hline $\begin{array}{l}\text { 1. Se visualiza futuro a través de criterios claros para } \\
\text { el éxito. }\end{array}$ & 3,38 & $\begin{array}{c}\text { Moderada presencia/Leve } \\
\text { fortaleza }\end{array}$ \\
$\begin{array}{l}\text { 2. Las decisiones se toman considerando hacia donde } \\
\text { se dirige la empresa financieramente en el futuro. }\end{array}$ & $\mathbf{2 , 4 0}$ & Baja presencia/Debilidad \\
\hline \begin{tabular}{l} 
Indicador \\
\hline
\end{tabular} & $\mathbf{2 , 8 9}$ & $\begin{array}{c}\text { Moderada presencia/Leve } \\
\text { fortaleza }\end{array}$ \\
\hline
\end{tabular}

Atendiendo estos resultados, a juicio del investigador, se ejecutan ciertas actividades basadas en lo que ha de ser financieramente las unidades de planes de salud de PDVSA Occidente en el futuro, señalando el rumbo a seguir, proporcionando su dirección financiera, coincidiendo con los señalamientos de Münch (2008), al referir la visión financiera como el enunciado del estado deseado en el futuro para la organización.

Continuando con el análisis del indicador: misión financiera se evidencia según la tabla 3 , un promedio de 2,16 ubicándolo en la categoría de baja presencia, señalando debilidad al afirmar los encuestados que los propósitos financieros generales establecidos son conocidos por 
todo el personal $(2,42)$ y que los propósitos financieros generales establecidos proporcionan criterios fundamentales para una adecuada inversión $(1,90)$.

\section{Tabla 3. Misión financiera}

\begin{tabular}{|c|c|c|}
\hline $\begin{array}{c}\text { Ítems } \\
\begin{array}{c}\text { En la unidad de planes de salud donde usted } \\
\text { labora: }\end{array}\end{array}$ & Media & Categoría \\
\hline $\begin{array}{l}\text { 3. Los propósitos financieros generales } \\
\text { establecidos son conocidos por todo el } \\
\text { personal. }\end{array}$ & 2,42 & Baja presencia/Debilidad \\
\hline $\begin{array}{l}\text { 4. Los propósitos financieros generales } \\
\text { establecidos proporcionan criterios } \\
\text { fundamentales para una adecuada }\end{array}$ & 1,90 & Baja presencia/Debilidad \\
\hline Indicador & 2,16 & Baja presencia/Debilidad \\
\hline
\end{tabular}

De manera que, en opinión del investigador, en las unidades de planes de salud de PDVSA Occidente, no se aplican usualmente actividades pertinentes que describan los propósitos financieros generales, por tanto no logran identificar a este elemento de la planificación financiera, situación discrepante con los señalamientos de Mitzberg y Quinn (2004), quienes describen, que los propósitos financieros generales establecidos por la organización, proporcionan criterios fundamentales al análisis de la adecuada inversión de la empresa a largo plazo.
Con relación al indicador valores financieros, se evidencia según la tabla 4 , un promedio de 3,10 ubicándolo en la categoría de moderada presencia, señalando leve fortaleza al afirmar los encuestados una moderada presencia respecto a que el personal se identifica con los valores financieros $(2,76)$ y alta presencia de que aplican herramientas motivadoras que permiten promover la productividad en la inversión realizada $(3,45)$.

\section{Tabla 4. Valores financieros}

\begin{tabular}{lcc}
\hline \multicolumn{1}{c}{ Ítems } & Media & Categoría \\
En la unidad de planes de salud donde usted labora: & 2,76 & $\begin{array}{c}\text { Moderada presencia/Leve } \\
\text { fortaleza }\end{array}$ \\
$\begin{array}{l}\text { 5. El personal se identifica con los valores financieros. } \\
\begin{array}{l}\text { 6. Aplican herramientas motivadoras que permiten } \\
\text { promover la productividad en la inversión } \\
\text { realizada. }\end{array}\end{array}$ 3,45 & Alta presencia/Fortaleza \\
\hline \begin{tabular}{l} 
Indicador \\
\hline
\end{tabular} & $\mathbf{3 , 1 1}$ & $\begin{array}{c}\text { Moderada presencia/Leve } \\
\text { fortaleza }\end{array}$ \\
\hline
\end{tabular}


En esta perspectiva, a criterio del investigador y según los resultados alcanzados, hay cierta presencia de acciones para describir los propósitos financieros generales establecidos por las unidades de planes de salud de PDVSA Occidente, y valores a los cuales sus trabajadores deben enfocar el esfuerzo de las finanzas de estas organizaciones; los mismos deben ser claros y precisos, estando en concordancia con Mitzberg y Quinn (2004), quienes señalan que los valores financieros son propósitos a los cuales la organización y sus miembros deben dedicar su energía; en tanto, la visión enmarca los propósitos financieros generales establecidos por la organización.
La tabla 5 recoge el comportamiento obtenido para el indicador objetivos financieros. Se observa un promedio de 2,88 lo cual según el baremo establecido lo ubica en la categoría de moderada presencia, señalando leve fortaleza, al considerar los encuestados con alta presencia que los objetivos financieros están en concordancia para la utilización de los recursos $(3,42)$; no obstante existe una debilidad por cuanto los lineamientos con baja presencia están enmarcados en la planificación de todas sus actividades financieras $(2,34)$.

Tabla 5. Objetivos financieros

\begin{tabular}{lccc}
\hline \multicolumn{1}{c}{ Ítems } & Media & Categoría \\
En la unidad de planes de salud donde usted labora: & 3,42 & Alta presencia/Fortaleza \\
$\begin{array}{l}\text { 7. Están los objetivos financieros en concordancia } \\
\text { para la utilización de los recursos. }\end{array}$ & 2,34 & Baja presencia/Debilidad \\
$\begin{array}{l}\text { 8. Los lineamientos están enmarcados en la } \\
\text { planificación de todas sus actividades financieras. }\end{array}$ & $\mathbf{2 , 8 8}$ & presencia/Leve fortaleza \\
\hline Indicador
\end{tabular}

Así las cosas, los resultados derivados a criterio del investigador demuestran una leve fortaleza en las unidades de planes de salud de PDVSA Occidente, en cuanto, a la manera de dirigir las actividades para aplicar de manera planificada los recursos financieros, situación inconveniente, en función de alcanzar el rendimiento apropiado en sus actividades financieras.

Las formulaciones descritas, son divergentes a los señalamientos de Cárdenas y Nápoles (2002), cuando consideran que los objetivos financieros son como los lineamientos de la institución enmarcados en planear y sistematizar todas las actividades del rendimiento sobre la inversión; por tanto, es un elemento relevante en la planificación estratégica financiera de las unidades de salud objeto de estudio, con el fin de establecer metas alcanzables.

En lo concerniente al indicador estrategias financieras, los resultados obtenidos se recogen en la tabla 6 , donde se aprecia una media de 3,73 ubicándolo en la categoría de alta presencia, señalando fortaleza al considerar los sujetos informantes que están definidos los pasos a seguir para el logro de los objetivos financieros $(3,67)$ y que se ejecutan estrategias financieras que muestran los 
esfuerzos requeridos para lograr los objetivos $(3,80)$.

Estos hallazgos le permiten afirmar al investigador que existen líneas de acción orientadas a alcanzar los objetivos financieros en las unidades de planes de salud de PDVSA Occidente, estos señalamientos concuerdan con Gitman, (2013) quien refiere que las estrategias financieras se definen como las acciones o pasos a seguir para el logro de los objetivos financieros $y$, por ende, necesita ser organizada, planeada, supervisada y dirigida, lo que orientará el sentido de la misma.

Tabla 6. Estrategias financieras

\begin{tabular}{|c|c|c|}
\hline $\begin{array}{l}\text { Ítems } \\
\text { En la unidad de planes de salud donde usted labora: }\end{array}$ & Media & Categoría \\
\hline $\begin{array}{l}\text { 9. Están definidos los pasos a seguir para el logro de } \\
\text { los objetivos financieros. }\end{array}$ & 3,67 & $\begin{array}{l}\text { Alta presencia/ } \\
\text { Fortaleza }\end{array}$ \\
\hline $\begin{array}{l}\text { 10. Se ejecutan estrategias financieras que muestran } \\
\text { los esfuerzos requeridos para lograr los objetivos }\end{array}$ & 3,80 & $\begin{array}{l}\text { Alta presencia/ } \\
\text { Fortaleza }\end{array}$ \\
\hline Indicador & 3,74 & $\begin{array}{l}\text { Alta presencia/ } \\
\text { Fortaleza }\end{array}$ \\
\hline
\end{tabular}

Cabe destacar, que estas acciones deben estar centradas en aspectos financieros que permitan guiar la acción de las unidades de planes de salud objeto de estudio para la consecución de sus objetivos financieros, sirve como base para lograr otros objetivos y ejecutar decisión; facilitan la toma de decisiones al evaluar alternativas, eligiendo aquella de la que se espera mejores resultados financieros.

Con respecto al indicador políticas financieras, según los resultados mostrados en la tabla 7 , se aprecia una media de 3,26 ubicándolo en la categoría de moderada presencia, señalando leve fortaleza, induciendo a afirmar a criterio de los sujetos informantes que con alta presencia se establecen una serie de principios de acción que permitan guiar el resultado financiero hacia el futuro $(3,55)$ y con moderada presencia consideran que las políticas financieras se adecuan al funcionamiento interno de acuerdo con las exigencias del entorno $(2,96)$.

Tabla 7. Políticas financieras

\begin{tabular}{|c|c|c|}
\hline $\begin{array}{c}\text { Ítems } \\
\text { En la unidad de planes de salud donde usted labora: }\end{array}$ & Media & Categoría \\
\hline $\begin{array}{l}\text { 11. Se establecen una serie de principios de acción que } \\
\text { permitan guiar el resultado financiero hacia el } \\
\text { futuro. }\end{array}$ & 3,55 & $\begin{array}{l}\text { Alta presencia/ } \\
\text { Fortaleza }\end{array}$ \\
\hline $\begin{array}{l}\text { 12. Las políticas financieras se adecuan al } \\
\text { funcionamiento interno de acuerdo con las } \\
\text { exigencias del entorno. }\end{array}$ & 2,96 & $\begin{array}{l}\text { Moderada presencia } \\
\text { /Leve fortaleza }\end{array}$ \\
\hline Indicador & 3,26 & $\begin{array}{c}\text { Moderada presencia/ } \\
\text { Leve fortaleza }\end{array}$ \\
\hline
\end{tabular}


La distribución de los datos descritos, refleja a criterio del investigador leves actividades para controlar aspectos de las finanzas en las unidades de planes de salud de PDVSA Occidente, que de continuar así sería contraproducente para la planificación de las acciones estratégicas financieras de estas organizaciones; ya que las políticas financieras deben estar dirigidas a controlar y mejorar determinados aspectos de las finanzas, según lo expuesto por Vera (2005), es este sentido las políticas financieras a emplear, deben estar a la par de otros elementos como la misión, visión, objetivo y valores de la organización; las mismas se deben adecuar a su funcionamiento interno de acuerdo con las exigencias del entorno para el logro de sus objetivos.

Respecto al indicador planes financieros se evidencia según la tabla 8 un promedio de 3,84 ubicándolo en la categoría de alta presencia, señalando fortaleza. Este resultado es producto al considerar los encuestados con alta presencia que se formulan planes financieros a objeto de precisar el desarrollo de sus actividades $(3,67)$ y se elaboran planes financieros para el desarrollo de nuevos servicios $(4,00)$.

Tabla 8. Planes financieros

\begin{tabular}{|c|c|c|}
\hline $\begin{array}{c}\text { Ítems } \\
\text { En la unidad de planes de salud donde usted labora: }\end{array}$ & Media & Categoría \\
\hline $\begin{array}{l}\text { 13. Formulan planes financieros a objeto de precisar el } \\
\text { desarrollo de sus actividades. }\end{array}$ & 3,67 & $\begin{array}{l}\text { Alta presencia/ } \\
\text { Fortaleza }\end{array}$ \\
\hline $\begin{array}{l}\text { 14. Elaboran planes financieros para el desarrollo de } \\
\text { nuevos servicios. }\end{array}$ & 4,00 & $\begin{array}{l}\text { Alta presencia/ } \\
\text { Fortaleza }\end{array}$ \\
\hline Indicador & 3,84 & $\begin{array}{l}\text { Alta presencia/ } \\
\text { Fortaleza }\end{array}$ \\
\hline
\end{tabular}

Los resultados obtenidos, a juicio del investigador demuestran una tendencia favorable en las unidades de planes de salud de PDVSA Occidente, por cuanto se tiende regularmente a elaborar planes financieros en función de incrementar el servicio ofrecido, lo cual es conveniente para estas unidades en la búsqueda de ofrecer un mejor servicio, que permitan garantizar el cumplimiento de objetivos previamente establecidos por la alta dirección, a propósito de preservar o mantener su posición en el mercado, coincidiendo estas argumentaciones con Perdomo (2002), al explicar que los planes financieros de desarrollo son tendientes a lograr objetivos de expansión, penetración de mercado, desarrollo de nuevos productos para el mismo mercado.

Para el indicador presupuesto, se evidencia según la tabla 9 un promedio de 4,27 ubicándolo en la categoría muy alta presencia, señalando fortaleza. Este resultado es producto al considerar los encuestados que con alta presencia se ejecuta la gestión funcional según el presupuesto formulado $(3,98)$ y con muy alta presencia se presentan en términos cuantitativos las actividades a ser realizadas por la unidad $(4,56)$. 


\section{Tabla 9. Presupuesto}

\begin{tabular}{|c|c|c|}
\hline $\begin{array}{c}\text { Ítems } \\
\text { En la unidad de planes de salud donde usted labora: }\end{array}$ & Media & Categoría \\
\hline $\begin{array}{l}\text { 15. Ejecuta la gestión funcional según el presupuesto } \\
\text { formulado. }\end{array}$ & 3,98 & $\begin{array}{l}\text { Alta presencia/ } \\
\text { Fortaleza }\end{array}$ \\
\hline $\begin{array}{l}\text { 16. Se presentan en términos cuantitativos las } \\
\text { actividades a ser realizadas por la unidad. }\end{array}$ & 4,56 & $\begin{array}{l}\text { Muy alta presencia/ } \\
\text { Fortaleza }\end{array}$ \\
\hline Indicador & 4,27 & $\begin{array}{l}\text { Muy alta presencia/ } \\
\text { Fortaleza }\end{array}$ \\
\hline
\end{tabular}

Visto así, a juicio del investigador, representa una fortaleza en la ejecución de acciones concernientes a presupuesto; evidenciando acciones financieras para operar adecuadamente en las unidades de planes de salud de PDVSA Occidente, por cuanto al conocer con certeza los recursos para llevar a cabo planes y actividades, siendo alentados el resultado a obtener. En ese sentido, las situaciones descritas concuerdan con Münch (2008), cuando explica que el presupuesto muestra la asignación de recursos para llevar a cabo los planes y actividades de la organización; también, con Cárdenas (2002), al referirlo como un instrumento de gestión para obtener el más óptimo uso de los recursos.

Como se puede apreciar en la tabla 10, se presenta el resumen para la dimensión elementos de la planificación estratégica, el cual muestra un valor de 3,27, reflejando de acuerdo a la opinión de los encuestados que existe una moderada presencia, señalando leve fortalezas en relación a las fases necesarias para la consecución de un plan, que regulen la vida de las finanzas a corto y largo plazo (Ross y otros, 2006) implicados o asociados en la cultura de la organización.

Estos resultados de moderada presencia que revelan leve fortaleza, pueden ser el inicio de los problemas diagnosticados al principio, cuando el investigador considero que el no contar con un plan estratégico para prever el actual escenario, desencadena una serie de situaciones no deseadas, las cuales, de no ser atendidas dentro de un esquema técnico científico, pueden llevar al colapso de los servicios de salud en las unidades de planes de salud de PDVSA Occidente. 
Tabla 10. Elementos de la planificación estratégica financiera

\begin{tabular}{lcc}
\hline \multicolumn{1}{c}{ Indicadores } & Media & Categoría \\
\hline Visión financiera & 2,88 & Moderada presencia/Leve fortaleza \\
Misión financiera & 2,16 & Baja presencia/Debilidad \\
Valores financieros & 3,11 & Moderada presencia/Leve fortaleza \\
Objetivos financieros & 2,88 & Moderada presencia/Leve fortaleza \\
Estrategias financieras & 3,74 & Alta presencia/Fortaleza \\
Políticas financieras & 3,26 & Moderada presencia/Leve fortaleza \\
Planes Financieros & 3,84 & Alta presencia/Fortaleza \\
Presupuesto & 4,27 & Muy alta presencia/Fortaleza \\
\hline \multicolumn{1}{c}{ Dimensión } & $\mathbf{3 , 2 7}$ & Moderada presencia/Leve fortaleza \\
\hline
\end{tabular}

En este sentido, para el investigador, conocer e implementar un mapa de ruta que marque el camino que habrá de tener el dinero dentro de las unidades de planes de salud objeto de estudio permite crear escenarios en donde se contemplen las diferentes realidades, y el abordaje adecuado que logre extraer el mayor beneficio posible, de allí la importancia de fortalecer los elementos de la planificación estratégica financiera, en consonancia con su estrategia general, para ello es importante descomponer una secuencia de fases necesarias para la consecución del plan (Perdomo, 2004).

\section{CONCLUSIONES}

En lo concerniente al objetivo, donde se identificó los elementos de la planificación estratégica financiera presentes en las unidades de planes de salud de PDVSA Occidente, se concluye una moderada presencia, señalando leve fortalezas en relación a las fases necesarias para la consecución de un plan.

En tal sentido se determinó que las actividades vinculadas al presupuesto, planes $y$ estrategias financieras constituyen fortalezas. Paralelamente se detectó pocas acciones vinculadas con la visión, valores, objetivos y políticas financieras en el proceso de planeación tomada en cuenta para el propósito de estas unidades, por lo que representan leve fortalezas, además, son ejecutadas irregularmente por las referidas unidades las actividades correspondientes a la misión financiera reportando una debilidad.

\section{REFERENCIAS}

Acedo, A. (2004). Rentabilidad, endeudamiento y costo de la deuda de las PYMES. Análisis empíricos de las empresas por tamaño. Revista Europea de Dirección y Economía de la empresa. España.

Betancourt, D. (2010), La Planificación Estratégica del Capital Humano en el siglo XXI. Editado por el Dpto. de Publicaciones de la Faculta de Ciencias Económicas y Sociales de la Universidad de Central de Venezuela, Caracas. Venezuela

Bruna, M. (2007). Contabilidad Estratégica. 1era. Edición. Editorial Editores. Argentina

Burbano, E. (2005), Presupuestos: Enfoque Moderno de Planeación y Control de 
Recursos. Tercera Edición. Mc Graw Hill. Bogotá. Colombia.

Cabreras, M. (2006). La planificación estratégica. Mc Graw Hill. México.

Campbell, N. y Nash, A. (2007), "Creating a Sense of Mission". Long Range Planning, Aug 91, Vol. 24 Issue 4, p10-20.

Catacora, J. (2008) Sistemas y Procedimientos Contables. Mc Graw Hill. Venezuela.

Chiavenato, I. (2009) Administración de recursos humanos. Quinta edición. Editorial Mc. Hill - Iberoamérica. Colombia.

Collins, O. y Porras, Y. (2007). Empresas que perduran. Norma. Bogotá. Colombia.

David, H. (2008). Comportamiento Humano en el trabajo. McGraw-Hill. España.

De Zayas, S. (2007) La Administración Financiera del Capital de Trabajo y la liquidez empresarial. Conceptos fundamentales. Hispano europea, España.

Francés, A., (2006). Estrategia y planes para la empresa con el Cuadro de Mando Integral. Primera Edición. México: Pearson Educación de México S.A. de C.V.

Galeano, P. y Tinjacá, J. (2012). Gerencia Financiera. San Martín: Fundación Universitaria San Martin. Argentina.

Gitman, L. (2013). Principios de administración financiera. Pearson Educación. México

Haime, L. (2013). El manejo estratégico de las finanzas para lograr el éxito empresarial. ISEF. Bogotá. Colombia.

López, V. (2012). Gestión Eficaz de los Procesos Productivos. Narcea. Madrid.

MacLeod, P. León, P. y Esquivias. P. (2011). Planificación estratégica y financiera integrada para organizaciones no gubernamentales. Usaid. EE.UU.

Magiolo, I. González, L. Bastidas, L. y Ferrer, B. (2014). Transformación de los servicios de salud mediante el dominio de la tecnología. Vol. 11 №.2 (2014) MarzoAgosto 2014
Mazón, L. Díaz, K. y Huerta, M. (2003). Administración organizacional. McGraw Hill. Barcelona. España

Mendoza, J. (2013). Decisiones estratégicas: macroadministración. Editorial Universidad del Norte. Barranquilla. Colombia

Mitzberg, H. y Quinn, J. (2004). La estructura de las organizaciones. Ariel Economía. Barcelona. España.

Münch, L. (2008). Visión financiera organizacional. McGraw Hill, 11‥ Trillas. México.

Ortega, J. (2008). Planificación organizacional. Editorial Mc. Hill. España

PDVSA página oficial de internet (201). Disponible en: http://www.pdvsa.com/index.php?tpl=in terface.sp/design/readmenuprinc_eps.tp I.html\&newsid_temas $=220$

Perdomo, C. (2004). Planificación financiera. Editorial Mc. Hill - Iberoamérica. Barcelona España

Ross, S.; Westerfield, R. y Jordan, B. (2006). Fundamentos de Finanzas Corporativas. Editorial Irwin. México

Serna, H. (2010). Gerencia estratégica. Teoría, metodología, alineamiento, implementación y mapas estratégicos, índices de gestión. Décima edición. Bogotá, Colombia.

Thompson, A. y Strickland, G. (2010). Administración Estratégica Conceptos y Casos 11a Edición. Ed. Mc. Graw Hill. New York. EE.UU.

Vera, M. (2005). EVA y CVA como medidas de la creación de valor de un negocio "Una introducción". Revista. Tendencias Volumen 1 No 1. Universidad de Nariño.

Welsch, P. Gordon, W. y Hilton, W. (2005). Presupuesto: Planificación y Control de Utilidades Editorial UTHEA. México.

Weston, F. (2006). Fundamentos de administración financiera. Edición 10a. Mc Graw Hill. México 